\title{
I Von der Modern Monetary Theory zur Forderung einer Jobgarantie
}

\author{
Maurice Höfgen ${ }^{\star}$ und Dirk Ehnts ${ }^{\star *}$
}

\section{Zusammenfassung}

Analytischer Ausgangspunkt der MMT ist die Tatsache, dass der Staat das Währungsmonopol besitzt. Dieses ermöglicht ihm, die zur Erfüllung der staatlichen Aufgaben benötigten Ressourcen aus dem Privatsektor zu mobilisieren. Über die Höhe der Staatsausgaben und die Höhe der Steuern entscheidet der Staat über das Ausmaß der unfreiwilligen Arbeitslosigkeit. Daraus lässt sich die Forderung nach einer staatlichen universellen Jobgarantie herleiten. Die ökonomischen Implikationen der Jobgarantie betreffen unter anderem die Konjunktursteuerung, die Preisstabilität, die Kaufkraft der Währung sowie die Außenwirtschaft. Die sozial-gesellschaftlichen Implikationen beziehen sich auf die sozialen und psychologischen Kosten, die mit unfreiwilliger Arbeitslosigkeit assoziiert werden, die Wirkungen auf die Arbeitsbedingungen im privaten Arbeitsmarkt, auf die mögliche Korrektur der Kräfteverteilungen zwischen ArbeitgeberInnen und ArbeitnehmerInnen sowie auf demokratierelevante Aspekte.

Schlagwörter: Modern Monetary Theory, Jobgarantie, Fiskalpolitik, Arbeitsmarkt

Deriving the Demand for a Job Guarantee from Modern Monetary Theory

Abstract

The analytical starting point of MMT is the fact that the state is the monopoly issuer of its currency. This enables the state to mobilize the resources from the private sector needed to fulfill the its public tasks. Through the level of government spending and taxation, the state decides on the extent of involuntary unemployment. From this insight, the demand for a universal job guarantee can be derived. The economic implications of the job guarantee relate to the management of the business cycle, price stability, the purchasing power of the currency and foreign trade. The social and societal implications relate to the social and psychological costs associated with involuntary unemployment, the effects on working conditions in the private labour market, a possible correction of the distribution of power between employers and employees and aspects relevant to democracy.

Keywords: Modern Monetary Theory, Job-Guarantee, Fiscal Policy, Labor Market

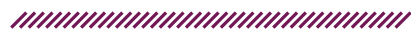

* Maurice Höfgen (Ansprechpartner), Samuel-Pufendorf-Gesellschaft für politische Ökonomie. E-Mail: Maurice.hoefgen@outlook.de.

** Dirk Ehnts, Samuel-Pufendorf-Gesellschaft für politische Ökonomie.E-Mail: dirk@ehnts.de.
} 


\section{Einleitung}

Die ökonomische Denkschule der Modern Monetary Theory, kurz: $M M T$, erhält sowohl im akademischen als auch im politischen Diskurs eine immer größere Aufmerksamkeit (WSJ Editorial Board 2020; Fullbrook/ Morgan 2020). Die Tatsache, dass die Bekämpfung der Coronakrise den Regierungen außergewöhnliche fiskalische Maßnahmen abverlangt hat, hat hierzu jüngst entsprechend beigetragen. Was die MMT von anderen Denkschulen unterscheidet, ist der analytische Ausgangspunkt. Dieser Ausgangspunkt ist bei der MMT die institutionelle Ausgestaltung des Geldsystems. Dadurch ermöglicht die MMT einen analytischen Blickwinkel, der auf der Funktionsweise moderner Geldsysteme basiert, auf die ökonomischen Fragen unserer Zeit. Dieser Blickwinkel führt $\mathrm{zu}$ wirtschaftspolitischen Schussfolgerungen, die anderen ökonomischen Denkschulen verwehrt bleiben. So lässt sich aus der MMT die wirtschaftspolitische Forderung nach einer universellen Jobgarantie, kurz: JG, ableiten, wie diese Arbeit theoretisch darlegen wird. Die JG ist dabei gewiss nicht nur von ökonomischer, sondern auch von sozial-gesellschaftlicher Bedeutung. Die ökonomische Herleitung und die sozial-gesellschaftlich motivierte Herleitung der Forderung einer JG sind dabei separat zu bewerten - auch wenn sie miteinander kompatibel sind und einer solchen wirtschaftspolitischen Forderung eine stärkere Argumentationsbasis verleihen. Das Ziel des Beitrags ist, die ökonomische Herleitung der JG aus den durch die MMT beschriebenen monetären Zusammenhänge moderner Volkswirtschaften verständlich herauszuarbeiten und eine kompakte Einführung in die ökonomischen und sozial-gesellschaftlichen Implikationen einer derartigen wirtschaftspolitischen Reform zu bieten.

Die Relevanz der Forderung nach einer JG wird mit Blick auf das Ausmaß an unfreiwilliger Arbeitslosigkeit von 7,4 Prozent und einer Jugendarbeitslosigkeit von 16 Prozent in der Eurozone (Stand: Juni 2020) deutlich - und durch die konjunkturellen Auswirkungen der Coronakrise auf Kurzarbeit, Unterbeschäftigung und Verfestigung von Langzeitarbeitslosigkeit gar noch verstärkt (Eurostat 2020; Bundesagentur für Arbeit 2020; AMS Arbeitsmarktservice 2020). Selbst in Deutschland, das im europäischen Vergleich eine relativ geringe Arbeitslosenquote aufweist, kamen im Jahr 2019 auf jede offene Stelle im Durchschnitt drei Arbeitssuchende. Die Quote fällt für andere Euroländer logischerweise entsprechend höher aus. Das führt dazu, dass unter den Arbeitssuchenden ein Verdrängungswettbewerb um knappe Arbeitsstellen herrscht (Institut Arbeit und Qualifikation der Universität Duisburg-Essen 2019). Neben den ökonomischen und sozialen Konsequenzen, die unfreiwillige Arbeitslosigkeit für die Betroffenen haben, stellt unfreiwillige Arbeitslosigkeit eine ineffiziente Ressourcennutzung dar (Tcherneva 2017b). Denn die Existenz unfreiwilliger Arbeitslosigkeit ist die Evidenz dafür, dass die Volkswirtschaft unterhalb ihres eigentlichen Potenzials operiert, also nicht alle verfügbaren produktiven Ressourcen ausgelastet werden, wodurch potenzielle Wohlstandsgewinne letztlich unrealisiert bleiben. Arbeit lässt sich nun mal nicht sparen: Wer ein Jahr lang gar nicht arbeitet, der kann nicht im nächsten Jahr doppelt so viel arbeiten und damit die „aufgesparte“ Arbeit wieder entsparen (Ehnts/Höfgen 2019a). Ferner hängt das Phänomen der unfreiwilligen Arbeitslosigkeit strukturell auch mit der Dominanz des Neoliberalismus zusammen. Wie Mitchell und Muysken (2008) feststellen, hat sich in der neoliberalen Ära das ökonomische Paradigma von der Vollbeschäftigung als Ziel der Wirtschaftspolitik abgekehrt und durch das Primat der Preisstabilität ersetzt. Ein gewisses Level an permanenter unfreiwilliger Arbeitslosigkeit wurde gar als Mittel zum Zweck der Preisstabilität degradiert bekannt unter dem englischen Akronym „NAIRU“ für „Non-Accelerating Inflationary Rate of Unemployment“, zu Deutsch: eine die Inflation nicht beschleunigende Arbeitslosenquote (Springer Gabler 2018). Dabei wird Arbeitslosigkeit als Instrument erachtet, mit dem Forderungen nach Lohnerhöhungen diszipliniert würden und eine dadurch beförderte inflationäre Lohn-Preis-Spirale verhindert würde. Vollbeschäftigung und Preisstabilität werden dementsprechend im neoliberalen Paradigma als Widerspruch gesehen (Galbraith 1997; Mitchell et al. 2019; Mitchell/Muysken 2008; Ehnts/Höfgen 2019a).

Der Beitrag wird wie folgt aufgebaut. Abschnitt 2 stellt die MMT und ihre analytischen Grundsätze und Implikationen vor. Abschnitt 3 schlägt dann die Brücke zur JG, leitet diese aus der MMT her und stellt das grundsätzliche Design einer JG vor. In Abschnitt 4 werden die ökonomischen Implikationen einer JG und in Abschnitt 5 die sozial-gesellschaftlichen Implikationen einer JG beschrieben. Abschnitt 5 liefert eine Zusammenfassung.

\section{Einführung in die MMT}

\subsection{Die Währung als Monopol des Staates}

Ausgangspunkt der MMT ist, dass der originäre Zweck des Geldsystems als das Ermöglichen einer adäquaten 
Bewirtschaftung der verfügbaren realen Ressourcen im Sinne des Gemeinwohls zu verstehen ist. Im Zentrum des Geldsystems steht die staatliche Fiat-Währung, die ein Monopol des Staates ist (Tcherneva 2002; Mosler 1997). Der Staat ist der einzige Schöpfer der Mittel, die er zur Zahlung von Steuern verlangt. ${ }^{1}$ So sind im Kontext der Eurozone lediglich die Europäische Zentralbank (EZB) und die ihr angeschlossenen nationalen Zentralbanken des Europäischen Systems der Zentralbanken (ESZB) berechtigt, den Euro in Form von elektronischen Eingaben im Zahlungssystem der Eurozone (TARGET2) zu erzeugen (Ehnts 202ob).

Ein Staat muss seine Währung erst einmal ausgeben oder in Form von Zentralbankkrediten verleihen und damit in Umlauf bringen, bevor nichtstaatliche Akteure die Währung zur Zahlung von Steuern oder zum Kauf von Anleihen verwenden können. Daraus folgt logischerweise, dass ein währungsherausgebender Staat zur Tätigung seiner Ausgaben weder auf Steuerzuflüsse noch auf den Verkauf von Staatsanleihen angewiesen ist - im Gegenteil: SteuerzahlerInnen und KäuferInnen von Staatsanleihen sind auf die Ausgabe der Währung angewiesen (Bell 2000; Bell 2001; Mosler/ Silipo 2017).

Ein Staat tätigt seine Ausgaben, indem er seine Zentralbank anweist, das Zentralbankkonto derjenigen Banken oder staatlichen Institutionen, an die die Zahlung adressiert ist, zu kreditieren. Das geschieht, bildlich gesprochen, indem die Zentralbank per Mausklick und Tastatureingabe das Konto des Empfängers erhöht (Fullwiler 2008; Fullwiler 2010). Aus technisch-funktionaler Sicht kann bei Staatsausgaben dementsprechend nur von „ausgeben“ und nicht von „finanzieren“ gesprochen werden. Das macht einen fundamentalen Unterschied, da der Begriff „finanzieren“ impliziert, dass es eine Mittelherkunft für staatliche Ausgaben geben muss. Das ist allerdings, wie erläutert, auf Ebene einer Bundesregierung nicht der Fall, da die Kontoerhöhungen ex nihilo, aus dem Nichts, vollzogen werden (Ehnts/Höfgen 2019b). Wie Wray (2015: 2) es ausdrückt: „[...] all dies war vor 200 Jahren offensichtlich, als Könige buchstäblich Münzen prägten, um sie auszugeben, und dann ihre eigenen Münzen als Steuerzahlung erhielten.“

1 Dasselbe gilt für andere an den Staat gerichtete Zahlungen. Da Steuern aber den größten Teil der an den Staat fließenden Zahlungen ausmachen, bleiben die anderen Zahlungen im Text unerwähnt, sind aber ebenso gemeint.
Man kann sich die Herausgabe der Währung ähnlich wie die Herausgabe von Kinotickets vorstellen. Das Kino erzeugt erst die Kinotickets und gibt diese an die KinobesucherInnen aus, bevor es die Tickets am Eintritt wieder einzieht und den BesucherInnen entsprechend Eintritt in das Kino gewährt. Erst kommt die Ausgabe der Währung (oder der Kinotickets), danach erst kann die Währung (die Tickets) wieder eingezogen werden. Ebenso wie das Kino verspricht, dass uns mit dem Kinoticket Eintritt gewährt wird, verspricht der Staat, dass er die herausgegebene Währung für unsere Steuerzahlungen akzeptiert. ${ }^{2}$ Daher kann vom Staat ausgegebenes Geld auch als Steuergutschrift verstanden werden (Ehnts/Paetz 2019).

\subsection{Die Bedeutung von Steuern}

Steuern erfüllen wichtige wirtschaftspolitische Funktionen, aber eben nicht die Finanzierung eines währungsherausgebenden Staates. 1945 hielt Beardsley Ruml, seinerzeit Vorsitzender der amerikanischen Zentralbankfiliale in New York, einen Vortrag mit dem Titel „Taxes for Revenue are obsolete“, zu Deutsch: Steuern zwecks Einnahmen sind überflüssig (Ruml 1946). Zu den wirtschaftspolitischen Funktionen, die Steuern erfüllen, zählen:

1) Steuern führen zur Akzeptanz der Währung durch die Staatsbevölkerung;

2) Steuern sind ein Instrument zur Steuerung der Gesamtnachfrage;

3) Steuern sind ein Instrument zur Korrektur von Ungleichheit;

4) Steuern beeinflussen Ausgabe- und Verhaltensentscheidungen.

Insbesondere die Bedeutung von Steuern für die Akzeptanz der Währung ist ein von der MMT viel beachteter Punkt, der auf die Ausführungen von Knapp (1905) und Innes (1914) zurückgeht. Beide erkannten, ohne sich der Arbeit des anderen wohl bewusst zu sein, dass der Wert der staatlichen Währung nicht mit intrinsischen Wertdeterminanten zusammenhängt, sondern mit der Tatsache, dass mit staatlicher Währung - und zwar nur damit - vom Staat auferlegte Steuerverpflichtungen getilgt werden können (Knapp 1905; Innes 1914).

2 Weitere Versprechen macht der Staat üblicherweise nicht. Er könnte aber einen festen Wechselkurs in Devisen (wie USD) oder in Unzen Feingold zumindest versprechen. Damit schränkt er sich allerdings freiwillig in seiner monetären Souveränität ein.
IIIOMENTUM QUARTERLY "IIIIIIIIIIIIIIIII 229 
Ein staatliches Defizit bedeutet also lediglich, dass der Staat mehr Steuergutschriften ausgibt, als er über Steuern und Abgaben einnimmt. Demnach entspricht die Staatsverschuldung den kumulierten Überschüssen an Steuergutschriften aus der Vergangenheit, die für zukünftige Steuerzahlungen oder für Ausgaben innerhalb des Privatsektors genutzt werden können (Mitchell et al. 2019; Wray 2015; Nersisyan/Wray 2016). ${ }^{3}$

$\mathrm{Da}$ jede/r StaatsbürgerIn in irgendeiner Form Steuerzahlungen (oder andere staatlichen Zahlungen) leisten muss, muss jede/r StaatsbürgerIn in Besitz der vom Staat herausgegebenen Währung gelangen. In Besitz der Währung gelangen WährungsnutzerInnen, indem sie dem Staat ihre Arbeit, Produkte oder Dienstleistungen gegen Bezahlung in staatlicher Währung verkaufen. Dies verdeutlicht die Motivation des Staates, eine eigene Währung herauszugeben: Der Staat ist zur Erfüllung seiner staatlichen Aufgaben auf die Arbeitskraft, Produkte oder Dienstleistungen seiner StaatsbürgerInnen angewiesen. Damit der Staat über diese verfügen kann, bezahlt er die StaatsbürgerInnen mit seiner eigenen Währung, welche er theoretisch unbegrenzt erzeugen kann. Um sicherzustellen, dass die StaatsbürgerInnen ihre Arbeitskraft, Produkte oder Dienstleistungen dem Staat auch anbieten, verlangt der Staat die Rückführung seiner eigenen Währung in Form von Steuerzahlungen. Die Steuer erzeugt also eine Nachfrage nach der staatlichen Währung (Mosler 1997; Tcherneva 2006). Die Währung wiederum ermöglicht dem Staat das Verfügen über die realen Ressourcen (Tcherneva 2017a; Forstater 2005). Da natürlich nicht jeder Akteur dem Staat seine Arbeit, Güter und Dienstleistungen verkaufen kann (will), werden auch Geschäfte des Privatsektors in staatlicher Währung abgewickelt, sodass auch diejenigen, die nicht direkt an den Staat verkaufen, an die zur Steuerzahlung nötigen staatlichen Währungseinheiten gelangen. Einzelne nichtstaatliche Akteure müssen also nicht unbedingt Geschäfte mit dem Staat abschließen, um an die Währung zu gelangen - der nichtstaatliche Sektor als Ganzes hingegen schon (Wray 2014).

\subsection{Wie tätigt ein Staat seine Ausgaben?}

In der heutigen Welt, in der die Zentralbank Zahlungen für das Finanzministerium leistet und ent-

3 Durch Letzteres wird Geld nur innerhalb des privaten Sektors hin- und hergeschoben. Wenn Steuern dabei anfallen, wird das Geld für Steuerzahlungen verwendet und verschwindet aus dem Kreislauf. gegennimmt, hat die Komplexität der operativen Prozesse zugenommen, doch die zugrunde liegende Logik der Ausgaben der Regierung bleibt die gleiche, die Wray (2015) mit der Praxis von KönigInnen, Münzen zwecks Ausgaben zu prägen und diese über Steuern wieder einzusammeln, ausdrückte. Anstatt Münzen zu prägen, tätigt das Finanzministerium seine Ausgaben, indem es seine Zentralbank anweist, den Betrag dem Zentralbankkonto der Geschäftsbank des jeweiligen Zahlungsempfängers gutzuschreiben. Die Geschäftsbank wiederum schreibt dann das Bankkonto ihrer KundInnen gut. Umgekehrt funktioniert es bei Steuerzahlungen. Die Zentralbank belastet das Zentralbankkonto der Geschäftsbank des Steuerzahlenden und diese belastet wiederum das Bankkonto ihres Kunden/ ihrer Kundin (Wray 2015; Ehnts 2020b; Fullwiler 2010). Wenn die Zentralbank einen bestimmten Interbankenzins anstrebt, was auf alle modernen Zentralbanken heute zutrifft, verkauft diese zudem Schuldinstrumente, in der Regel Staatsanleihen, an den Bankensektor, um die überschüssigen Reserven, die sich aus den Staatsausgaben, also dem Erhöhen der Zentralbankkonten der Banken, ergeben, abzuschöpfen andernfalls führten Staatsausgaben zu einem temporären Überschuss an Reserven, die den Zinssatz nach unten, in Richtung null oder in Richtung des Einlagezinses, drücken und das Interbankenzinsziel der Zentralbank gefährden würden. Staatsanleihen sind damit für die Geldpolitik ein Instrument zur Zinssteuerung (Fullwiler 2006; Forstater/Mosler 2005; Bell/Wray 2002).

Diese Absorption von überschüssigen Reserven durch Offenmarktgeschäfte der Zentralbank zeigt, dass der Verkauf von Staatsanleihen für währungsemittierende Staaten nur ein geldpolitisches Instrument zur Zinssteuerung und kein fiskalpolitisches Instrument zur Geldbeschaffung ist (Fullwiler 2011; Hail 2018). Zwar gibt es in den einzelnen Ländern unterschiedliche operative und institutionelle Verfahren für die horizontale Beziehung zwischen dem Finanzministerium und seiner Zentralbank - verankert in der jeweiligen Geldverfassung -, doch diese sind in den meisten Fällen für die vertikale Beziehung zwischen dem konsolidierten Staatssektor (Finanzministerium plus Zentralbank) und dem nichtstaatlichen Sektor weitgehend irrelevant (Tymoigne/Wray 2015; Tymoigne 2014; Fullwiler et al. 2012). Ob eine Regierung ihr Staatskonto bei der Zentralbank überziehen kann, ob sie Staatsanleihen direkt an die Zentralbank verkaufen darf (wie z. B. in Kanada) oder ob sie die Staatsanleihen nur an Geschäftsbanken 
verkaufen darf, die allerdings dafür von der Zentralbank zu vorgegebenen Bedingungen jederzeit mit ausreichend Reserven ausgestattet werden (wie z. B. in Deutschland), macht funktional keinen Unterschied. Die Bilanzen der beteiligten Akteure sehen am Ende des Ausgabenprozesses nahezu identisch aus (Ehnts 2020a; Höfgen 2020; Wray 2015). Alle freiwilligen, selbst auferlegten Verfahrensvorschriften, die die Regierung in ihrer Ausgabefähigkeit einschränken, sind für währungsausgebende Regierungen als ökonomisch unnötig zu betrachten und können nur politisch begründet werden.

\subsection{Der Unterschied zwischen Währungsnutzern und Währungsherausgeber}

Als Währungsherausgeber operiert der Staat nach einer völlig anderen Logik als die Nutzer einer Währung, wie etwa Kommunen, Unternehmen oder Haushalte. Währungsnutzer müssen ihre Ausgaben entweder durch Einkommen, Vermögensverkäufe oder Kreditaufnahme (begrenzt durch ihre Kreditwürdigkeit) finanzieren. Das trifft auf den Währungsherausgeber nicht $\mathrm{zu}$, denn dieser kann alle Zahlungen, die in eigener Währung notiert sind, bei entsprechender Fälligkeit leisten und hat kein Insolvenzrisiko bei Schulden, die auf eigener Währung lauten. Ein währungsherausgebender Staat ist daher in der Lage, alles, was in eigener Währung zum Verkauf angeboten wird, in seiner eigenen Währung zu kaufen - einschließlich aller von den BürgerInnen angebotenen Arbeitskraft (Wray 2015; Tcherneva 2002; Tymoigne 2019). Im Gegensatz zum Nutzer einer Währung unterliegt der Herausgeber also keinen finanziellen Grenzen. Die einzigen Einschränkungen, denen eine monetär souveräne Regierung gegenübersteht, sind die Verfügbarkeit und die Qualität ihrer realen Ressourcen sowie das Risiko, nachfrageseitigen Inflationsdruck $\mathrm{zu}$ erzeugen, wenn die Gesamtausgaben die Produktionskapazität der Wirtschaft übersteigen (Kelton 2011). Die relevanten Kosten der Fiskalpolitik sind für einen Währungsherausgeber nicht monetärer Natur. Vielmehr sind jene Kosten als Opportunitätskosten für die Nutzung der endlichen realen Ressourcen zu sehen. Daraus folgt, dass die Fiskalpolitik nicht auf monetäre Buchhaltungsergebnisse, wie etwa einen bestimmten Haushaltssaldo am Ende einer Wirtschaftsperiode, sondern auf funktionale Ziele, wie etwa Vollbeschäftigung oder ökologische Indikatoren, ausgerichtet werden sollte (Ehnts/Höfgen 2019a; Hail 2018; Kelton 2020).

\subsection{Das Spektrum monetärer Souveränität}

Dennoch können auch Währungsherausgeber wirtschaftspolitisch ihre eigenen Hände in Ketten legen. Dies ist beispielsweise der Fall, wenn die Regierung eine feste Wechselkursstrategie verfolgt, also verspricht, ihre Währung zu einem bestimmten Kurs in eine Fremdwährung umzutauschen oder sogar Edelmetall, wie in den diversen Goldstandardregimen im 20. Jahrhundert üblich, zu einem festen Kurs anzubieten. Während einer monetär souveränen Regierung zwar nicht das eigene Geld ausgehen kann, können ihr jedoch die Devisenreserven oder die jeweiligen Edelmetallvorräte ausgehen und sie zwingen, den Wechselkurs aufzugeben (Mitchell/Fazi 2017; Wray/Sardoni 2007; Mosler/ Forstater 1999). Der Grad an monetärer Souveränität eines Staates hängt dabei im Wesentlichen von vier Faktoren ab:

1) Der Staat gibt seine eigene Währung aus;

2) Der Staat ist in der Lage, Steuern in der eigenen Währung einzutreiben;

3) Der Staat geht keine Fremdwährungsverschuldung ein;

4) Der Staat gibt kein Versprechen ab, die eigene Währung zu einem fixen Wechselkurs in eine andere Währung oder bestimmte Edelmetalle zu tauschen.

Je höher der Grad monetärer Souveränität eines Staates, desto größer der wirtschaftspolitische Handlungsspielraum. Andersherum: Je geringer der Grad an monetärer Souveränität, desto kleiner der wirtschaftspolitische Handlungsspielraum und desto größer die Notwendigkeit politischer Kompromisse (Wray 2015; Tymoigne 2019). Wofür dieser genutzt wird, ist dann in der Regel von politischen Überzeugungen der Regierenden abhängig. Die MMT ist kein politisches Regime, sondern als analytische Linse zu verstehen, aus der alle Volkswirtschaften dieser Welt analysiert werden können. Ausgangspunkt hierbei ist, wie gesagt, die institutionelle Ausgestaltung des Geldsystems. Daher sind Argumente, dass die MMT beispielsweise nur auf den Fall der USA zutreffe, zurückzuweisen.

\section{Die Jobgarantie als logische Schlussfolgerung aus der MMT}

Die Idee der Jobgarantie kann aus den Einsichten der MMT zur Funktionsweise des Geldsystems gefolgert werden. Nach der MMT wird der originäre Zweck des Geldsystems als das Ermöglichen einer adäquaten Bewirtschaftung der verfügbaren realen Ressourcen
MOMENTUM QUARTERLY "IIIIIIIIIIIIIIII 231 
im Sinne des Gemeinwohls verstanden (Mosler 1997; Murray/Forstater 2013b; Ehnts/Höfgen 2020). Im Zentrum des Geldsystems steht die staatliche Währung. Das Nutzen der staatlichen Währung, deren Herausgabe dem (monetär) souveränen Staat obliegt, ermöglicht diesem das Mobilisieren der zur Erfüllung der staatlichen Aufgaben notwendigen Ressourcen, etwa der Arbeitskraft seiner StaatsbürgerInnen. Durch das Auferlegen einer Steuerpflicht schafft der Staat eine Nachfrage nach seiner Währung, denn die steuerpflichtigen Währungsnutzer müssen zur Erfüllung der Steuerpflicht an die vom Staat herausgegebene Währung gelangen. Dies gelingt ihnen, indem sie ihre Arbeitskraft, Güter oder Dienstleistungen gegen Bezahlung in staatlicher Währung, die ausschließlich und unbegrenzt vom Staat geschöpft wird, anbieten (Tcherneva 2002; Tcherneva 2006). Damit ist staatliche Währung quasi eine Marktlösung für das Problem der Versorgung mit realen Ressourcen des Staates.

Dieser Logik folgend entsteht unfreiwillige Arbeitslosigkeit - definiert als Personen, die nach Arbeit suchen, für die sie in der staatlichen Währung bezahlt werden -, wenn der/die WährungsnutzerIn den eigenen Wunsch, die staatliche Währung zu akkumulieren, nicht ausreichend erfüllt wird. Das gilt, solange es Personen gibt, die in staatlicher Währung bezahlte Arbeit suchen, um an die herausgegebene Währung zu gelangen (Mosler 1997). Mit anderen Worten: Unfreiwillige Arbeitslosigkeit ist eine wirtschaftspolitische Entscheidung des Staates und folglich der ultimative Beweis dafür, dass die Staatsausgaben nicht hoch genug sind - entweder ist der Staatsüberschuss zu groß oder das Staatsdefizit zu gering (Mitchell/Muysken 2008). Die Ursache liegt also aus makroökonomischer Sicht in der Fiskalpolitik des Staates begründet.

Um dieser Verantwortung gerecht zu werden, kann der Staat über eine Jobgarantie die Staatsausgaben um genau die Menge erhöhen, die zur Befriedigung des Wunsches des Privatsektors, die staatliche Währung zu akkumulieren, sowie zur Erreichung von Vollbeschäftigung nötig ist. Dazu macht der Staat ein universelles Jobangebot an jeden/jede, der/die zu einem sozialverträglichen Lohn inklusive Lohnnebenleistungen arbeiten möchte (Ehnts/Höfgen 2019a; Wray et al. 2018; Tcherneva 2018). Die Jobgarantie kann dabei auf Ebene der Bundesregierung finanziert und von unteren Regierungsebenen, etwa auf Stadt- oder Gemeindeebene, verwaltet werden. Dadurch würde der individuelle Wunsch nach kontinuierlicher Beschäftigung mit den Bedürfnissen von Städten und Gemeinden in Sachen öffentlicher
Daseinsvorsorge kombiniert. Passende Arbeitsbereiche für die Jobgarantie wären gemeinwohlorientierte Beschäftigungen, die auf die Verbesserung des Zustands der Stadt bzw. der Gemeinde ausgerichtet sind, sowie jene, die vom Privatsektor nur unzureichend abgedeckt werden. Konkret könnte die Jobgarantie Projekte aus den Bereichen Bildung, Ausbildung, Pflege, Kunst, Umweltmanagement, Stadtpflege, lokale Lebensmittelproduktion oder Sicherheit beinhalten. Hinsichtlich der Art der Beschäftigungsverhältnisse ist die Jobgarantie nicht auf Vollzeitstellen begrenzt, sondern kann auch Teilzeitstellen sowie flexible, andersgeartete Arbeitsverhältnisse beinhalten, um möglichst vielen Lebenssituationen zu entsprechen - von der/dem Studierenden bis zum Elternteil (Tcherneva 2018; Kaboub 2006).

Wie die MMT aufdeckt, ist die Frage nach der Finanzierbarkeit oder den finanziellen Kosten einer wirtschaftspolitischen Maßnahme im Kontext von Staaten mit einem ausreichenden Grad an monetärer Souveränität nicht zielführend. Ein solcher Staat hat keine finanziellen Grenzen und kann sich jedes Projekt finanziell „leisten“ - unabhängig davon, wie hoch die monetären „Kosten“ sind. Die tatsächlichen Kosten sind die realen Güter und Dienstleistungen, die innerhalb der JG Verwaltung benötigt und von JG-TeilnehmerInnen durch deren zusätzliche Einkommen konsumiert werden (Ehnts/Höfgen 2019a; Höfgen 2020).

Bei Staaten ohne einen solchen Grad an monetärer Souveränität, wie z. B. den Ländern der Eurozone, wird die Finanzierung zur Frage von politischer Priorität und hat bei der aktuellen institutionellen Ausgestaltung, insbesondere der fiskalpolitischen Regelungen, der Eurozone unter Umständen auch finanzielle Opportunitätskosten. Im Vergleich mit den fiskalischen Kosten von Langzeitarbeitslosen kommen Tamesberger und Theurl (2019) für eine Jobgarantie für Langzeitarbeitslose in Österreich bei einem Bruttogehalt von 27.000 Euro pro Person und Jahr und unter Berücksichtigung von Rückflüssen durch Verbrauchssteuern und Sozialversicherungsbeiträgen auf Mehrkosten von 6.785 Euro pro Person und Jahr. Doch auch hier gilt: Die EZB könnte die notwendigen Mittel in beliebiger Höhe auf Knopfdruck erzeugen. Die derzeitige institutionelle Ausgestaltung der Eurozone steht dem allerdings im Wege. Die Hürden sind also politischer, nicht ökonomischer Natur. Wenn bei den nationalen Regierungen der politische Wille zur Verfolgung einer Vollbeschäftigungspolitik bestünde, aber die institutionelle Ausgestaltung des Geldsystems den Regierungen die Hände bindet, dann kann die Schlussfolgerung nur sein, dass diese institutionelle 
Ausgestaltung des Geldsystems zu hinterfragen und zu verändern ist. Das kann unter anderem das Reformieren oder gar Verlassen der Währungsunion bzw. in anderen Kontexten das Aufgeben einer fixen Wechselkursstrategie bedeuten.

Ehnts, Tcherneva und Cruz-Hidalgo (2019) machen im Kontext der Eurozone den Vorschlag, die Umsetzung einer JG durch die Einführung eines europäischen Finanzministeriums zu ermöglichen. Während die Mitgliedsländer durch Aufgabe der eigenen Währung zu Währungsnutzern wurden und damit finanzpolitischen Handlungsspielraum aufgegeben haben, wurde beim Design der Währungsunion verpasst, den Wegfall an Handlungsspielraum mit einer fiskalischen Institution auf supranationaler Ebene zu schaffen. Das könnte mit einem europäischen Finanzministerium korrigiert werden. Wie weit das Mandat eines solchen Finanzministeriums gespannt werden soll, ist eine politische Frage. Ehnts, Tcherneva und Cruz-Hidalgo (2019) plädieren dafür, dass die Finanzierung der JG der kleinste gemeinsame Nenner der Euroländer sein sollte. Dabei sollte das Finanzministerium, wie etwa Finanzministerien anderer Länder mit monetärer Souveränität, eigene Anleihen („Eurobonds") herausgeben dürfen. Die Anleihen würden - ebenso wie jene der Mitgliedsstaaten derzeit - auf dem Primärmarkt an Banken verkauft, die dafür Zentralbankguthaben bezahlen. Die EZB könnte erklären, im Zweifel alle europäischen Anleihen über Käufe auf dem Sekundärmarkt in die eigene Bilanz zu nehmen. Damit wäre die ohnehin mit hoher Bonität versehenen Anleihen de facto risikolos. Die Ausgaben des Finanzministeriums wären dann abhängig von der Größe der nationalen JG-Programme bzw. der Höhe der unfreiwilligen Arbeitslosigkeit. Damit könnte auch trotz zu eng geschnürter Fiskalregeln für die Mitgliedsländer dauerhafte Vollbeschäftigung erzielt werden. Die Defizite eines europäischen Finanzministeriums bedeuteten zusätzliche Einkommen für die Haushalte der nationalen Regierungen und würden die angespannte fiskalische Situation in einigen Euroländern entspannen (Ehnts 2020b; Höfgen 2020).

\section{Zu den ökonomischen Implikationen einer Jobgarantie}

4.1 Die Jobgarantie als Mittel zur Konjunktursteuerung

Die JG wirkt konjunkturell als automatischer antizyklischer Stabilisator. Im wirtschaftlichen Abschwung erhöht sich die Zahl an JG-TeilnehmerInnen und auf diese Weise die damit verbundenen Staatsausgaben, während im wirtschaftlichen Aufschwung die Zahl an JG-TeilnehmerInnen und folglich die damit verbundenen Staatsausgaben zurückgehen. Gerade während einer Rezession sind die zusätzlichen Staatsausgaben genau der Stimulus, den es zur Erholung vom wirtschaftlichen Tief benötigt (Tcherneva 2018; Wray 1997). In jener Hinsicht ist die JG mit dem Konzept des Arbeitslosengeldes, welches den Verlust an Einkommen zwecks Stabilisierung der Nachfrage während einer Rezession kompensiert, vergleichbar - mit einem entscheidenden Unterschied allerdings: Die JG kompensiert im Gegensatz zum Arbeitslosengeld nicht nur den Einkommens-, sondern auch den Beschäftigungsverlust. Dazu ist die Änderung des Konsumverhaltens kleiner, wenn Personen keinen Beschäftigungsverlust erfahren. Das gilt insbesondere für Betroffene aus Ländern, in denen die Arbeitslosengeldzahlungen etwa nur zeitlich befristet sind (Tcherneva 2017b).

Die zentrale Einsicht der MMT, dass sich ein Staat mit ausreichender monetärer Souveränität keinen finanziellen, sondern nur realen Ressourceneinschränkungen ausgesetzt sieht, hat das Potenzial, den Fokus der Fiskalpolitik grundlegend zu verändern. Im aktuellen Narrativ, in welchem der Fokus auf dem rein finanziellen fiskalpolitischen Handlungsspielraum liegt, ist es unwichtig, woher die Gelder stammen, um Ausgaben zu „finanzieren“. Sobald aber die Grenze des fiskalischen Handlungsspielraums in der Verfügbarkeit produktiver Ressourcen verortet wird, rückt eben das bloße Geldauftreiben im Gegensatz zu einer detaillierten Betrachtung des Auslastungsgrades realer Ressourcen in den Hintergrund. Um durch das Freisetzen realer Ressourcen, etwa durch Steuern oder strengere Regulierungen, fiskalischen Spielraum zu schaffen, ist es unabdingbar für die Fiskalpolitik, branchen- und regionsspezifisch zu analysieren (Fullwiler et al. 2019). Es hilft beispielsweise nicht, wenn zur Umsetzung eines wirtschaftspolitischen Projektes Arbeitskräfte im Norden des Landes freigesetzt, um im Süden des Landes eingesetzt zu werden. Das Ziel sollte sein, eine über alle Regionen gleichmäßig wirkende Wirtschaftsentwicklung zu erzielen. Die Höhe der JGBeschäftigten kann dabei ein relevanter Indikator für den branchen- und regionsspezifischen Auslastungsgrad der Wirtschaft sein, auf den die Wirtschaftspolitik ausgerichtet werden kann (Kelton 2020).

Ferner baut die JG auf der Einsicht auf, dass die gesamtwirtschaftliche Nachfragesteuerung eine zentrale Aufgabe staatlicher Wirtschaftspolitik ist und 
nicht allein dem Privatsektor überlassen werden kann. Ein vom Privatsektor angeführter Wirtschaftsstimulus führt zum Aufbau von privaten Verschuldungspositionen und damit zu einer fragileren Finanzlage des währungsnutzenden Privatsektors, während die fiskalpolitische Nachfragestimulierung über die JG zum Nettogeldvermögensaufbau im nichtstaatlichen Sektor führt und damit dessen finanztechnische Lage verbessert. Wie die MMT verdeutlicht, agieren Währungsherausgeber und Währungsnutzer unter gänzlich verschiedenen Voraussetzungen. Da Währungsnutzer im Gegensatz zu Währungsherausgebern finanziellen Einschränkungen unterliegen, ist ein fiskalpolitisch induzierter Wirtschaftsaufschwung vor dem Hintergrund finanzieller Nachhaltigkeit dem privat angeführten Anschub der Wirtschaft überlegen (Kelton 2011; Nersisyan/Wray 2016). Das bedeutet allerdings nicht, dass angebotsseitige Maßnahmen wie etwa Anreize für private Investitionen oder auch eine nachfrageorientierte Lohnpolitik an Bedeutung für die Steuerung der Konjunktur verlieren.

4.2 Preisstabilität und die Abkehr von der NAIRU

Im Vorfeld des neoliberalen Zeitalters war Vollbeschäftigung ein explizites wirtschaftspolitisches Ziel, auf das Fiskal- und Geldpolitik entsprechend zugeschnitten wurden (Mitchell/Muysken 2008; Palley 2005). Danach ist das Ziel der Preisstabilität zum primären Ziel der Wirtschaftspolitik geworden, dem die Vollbeschäftigung als Ziel untergeordnet wurde. In einer Wirtschaftspolitik, die auf das Konzept der NAIRU baut, wird Arbeitslosigkeit genutzt, um Forderungen nach Lohnerhöhungen der abhängig Beschäftigten zu disziplinieren und eine damit verbundene inflationäre Lohn-Preis-Spirale zu verhindern. Nach dem Ansatz werden, sobald die inflationsstabile Arbeitslosenquote erreicht wird, wirtschaftspolitische Maßnahmen getroffen, um einen weiteren Rückgang der Arbeitslosigkeit zu verhindern. Vollbeschäftigung und Preisstabilität werden dementsprechend als Widerspruch gesehen. Während der zurzeit verfolgte NAIRU-Ansatz einen Pufferbestand an unfreiwillig Arbeitslosen zwecks Preisstabilität vorsieht, baut die JG auf einen Pufferbestand an Beschäftigten auf, die zu einem festen Lohn jederzeit Anstellung finden können. Mitchell und Muysken (2008) bezeichnen die Alternative zur NAIRU als „NAIBER“, was für „Non-Accelerating Inflation Buffer Employment Ratio“ steht - zu Deutsch: eine Inflation nicht beschleunigenden Pufferbestand-
Beschäftigungsquote. Die NAIBER beschreibt also im Gegensatz zur NAIRU nicht das Verhältnis von Beschäftigten zu Arbeitslosen, sondern von Beschäftigten zu JG-TeilnehmerInnen.

Auch wenn die JG nicht dazu konzipiert ist, alle Ursachen von Inflation bzw. Deflation zu adressieren, hilft die Stabilisierung des Konjunkturzyklus dabei, sowohl inflationäre als auch deflationäre Tendenzen abzumildern. Der fixe Stundenlohn, den alle JGTeilnehmerInnen erhalten, wirkt dabei als effektiver Preis- und Lohnanker (Wray 1997; Mosler/Silipo 2017). Als alleiniger Herausgeber der Währung hat der Staat dieselbe Preisgestaltungsmacht wie andere Monopolisten. Mit der JG macht der Staat davon Gebrauch und legt den Mindestpreis für eine Stunde Arbeit in Austausch für die herausgebende Währung fest. Alle anderen Preise passen sich gemäß der Marktmechanismen an und werden letztlich durch Angebot, Nachfrage und Marktmacht bestimmt. Jedoch spiegeln diese nun den relativen Wert zum Preis von einer Arbeitsstunde innerhalb der JG wider (Mosler/Forstater 1999; Mosler 1997; Tcherneva 2002). Im Vergleich zum bisherigen NAIRU-Ansatz, der auf einen Pufferbestand an Arbeitslosen baut, macht dieser Ankermechanismus den JG-Ansatz zum überlegenen Mittel zur Erreichung von Preisstabilität. Darüber hinaus wirkt die JG hemmend auf inflationäre Tendenzen, indem ArbeitnehmerInnen der Wechsel in den Privatsektor erleichtert wird. Üblicherweise bevorzugen Unternehmen bei der Einstellung Personen, die vorher nicht arbeitslos waren, da diese während ihrer Anstellung betriebliche Weiterbildung erfahren und Leistungsbereitschaft demonstriert haben, was auch bedeutet, dass Langzeitarbeitslose für Unternehmen in dem Kontext am unattraktivsten sind (Mosler 2010). Da die JG OnThe-Job-Training beinhaltet und den TeilnehmerInnen das Demonstrieren von Leistungsbereitschaft, Pünktlichkeit, Teamfähigkeit etc. abverlangt, erleichtert die JG für Firmen die Mitarbeitersuche und verringert die damit verbundenen Kosten und den Zeitaufwand. Das hat zur Folge, dass Firmen ihre Produktionskapazitäten in Fällen, in denen die Gesamtnachfrage die bestehenden Kapazitäten übersteigt, schneller ausweiten können. Das schnellere Ausweiten der Produktionskapazität ermöglicht den Firmen, auf erhöhte Nachfrage mit einer Ausweitung der Absatzmenge - statt mit Preiserhöhungen - zu reagieren und reduziert somit das Risiko einer nachfrageseitig induzierten Inflation. Man könnte auch sagen: Der NAIBER-Puffer ist liquider als der NAIRU-Puffer und damit ein effizienteres 
Mittel zur Stabilisierung des Preislevels (Mosler 2010; Tcherneva 2018; Tcherneva 2012).

Im Vergleich zur typisch keynesianischen Wirtschaftsankurbelung mittels Erhöhung der Staatsausgaben ohne explizite Schaffung von staatlichen Beschäftigungsmöglichkeiten, dem sogenannten „pump-priming“, bei dem der Staat mit seinen Ausgaben um Güter und Dienstleistungen zu Marktpreisen konkurriert, werden die JG-Ausgaben dorthin gelenkt, wo es im Moment keine Nachfrage des Privatsektors nach den verfügbaren Arbeitskräften gibt, sodass durch Wettbieten ausgelöste Preisanstiege vermieden werden. Darüber hinaus ist die JG dem stumpfen Erhöhen der Staatsausgaben überlegen, da der klassische Weg keinen Anti-Inflations-Mechanismus enthält, keine regionalen Disparitäten adressiert und keine ausdrückliche Orientierung am Gemeinwohl sicherstellt, da ohne JG letztlich die Unternehmen entscheiden, wo und welche Jobs geschaffen werden. Im Gegensatz zur JG kommt das „pump-priming“ bei den Personen, die im Boom als Letzte Anstellung finden und in der Rezession als Erste entlassen werden, nur sehr bedingt an (Ehnts/Höfgen 2019a; Mitchell/Muysken 2008).

\subsection{Anzunehmende Preis- und Beschäftigungseffekte bei Einführung der Jobgarantie}

Abhängig von der gewählten Höhe des Stundenlohns bei Einführung der JG, löst dieser unter Umständen eine einmalige Preisanpassung sowie einen Beschäftigungseffekt im Privatsektor aus. Wenn der JG-Lohn $\mathrm{zu}$ hoch angesetzt wird, dann wechseln ArbeitnehmerInnen vom Privatsektor in das JG-Programm, was wahrscheinlich aufgrund des steigenden Einkommens sowie der erhöhten Lohnkosten für die Unternehmen im Privatsektor (sowohl um abwanderungswillige ArbeitnehmerInnen zu halten als auch um neue ArbeitnehmerInnen $\mathrm{zu}$ rekrutieren) $\mathrm{zu}$ einem einmaligen Preis- und Lohnanstieg führt (Mosler 1997; Murray/ Forstater 2013b). Allerdings ist es in der aktuellen Lage, in der die meisten Volkswirtschaften unterausgelastet sind, zu erwarten, dass Unternehmen auf zusätzliche Nachfrage mit einer Ausweitung der Produktion statt mit Preiserhöhungen reagieren. Zusätzlich ist anzunehmen, dass der anfängliche Einkommensschub von den EmpfängerInnen erst einmal für Schuldentilgungen oder das Aufbauen von Rücklagen genutzt wird (Ehnts/ Höfgen 2019a). Wird der anfängliche JG-Lohn jedoch zu niedrig angesetzt, würde dies tendenziell zu einem einmaligen Effekt der Preissenkung führen. Das liegt darin begründet, dass dann das Einkommen des Privatsektors nicht ausreicht, um die gewünschte Summe an herausgegebener Währung zu akkumulieren und die gesamte Produktionsmenge der Unternehmen abzusetzen, was zu einer Erhöhung der Lagerbestände bei den Unternehmen sowie zu Entlassungen und einem dadurch ausgelösten abwärtsgerichteten Druck auf Löhne und Preise führt. Da der JG-Lohn jedoch nicht an irgendeinen Preisindex und damit an die Entwicklung anderer Preise gekoppelt ist, beinhaltet die JG keinen internen Mechanismus, der die LohnPreis-Spirale triggert (Mosler 1997). Die durch die Implementierung der JG ausgelösten Preiseffekte sind von einmaliger Natur und resultieren nicht in Inflation bzw. Deflation, was als kontinuierlicher Anstieg bzw. Fall des Preislevels definiert ist (Mitchell et al. 2019).

\subsection{Zur Kaufkraft der Währung}

Die JG sorgt ferner dafür, dass der Währung eine explizite Kaufkraft beigemessen werden kann. Die Kaufkraft der Währung wird davon beeinflusst, was der Währungsherausgeber von den WährungsnutzerInnen zwecks Erhalts der Währung verlangt - dies betrifft das Erbringen von Arbeitsleistungen als auch den Verkauf von Gütern und Dienstleistungen. Die JG definiert die Kaufkraft der Währung, indem sie festlegt, welche Menge und welche Qualität an Arbeitskraft für welchen Preis (Lohn) bezogen werden kann (Mosler 1998; Mosler 1997). Dieser Logik folgend stärken Maßnahmen innerhalb der JG, die die Weiterbildung als auch den Aufbau neuer Fertigkeiten fördern, die Kaufkraft der Währung, indem die Qualität der Arbeitskraft, die zum JG-Lohn bezogen werden kann, verbessert wird (Tcherneva 2018; Ehnts/Höfgen 2019a).

\subsection{Außenwirtschaftliche Implikationen}

In einer globalisierten Welt mit vernetzten Märkten ist natürlich auch die Frage wichtig, welche Auswirkungen die JG auf den Handel mit anderen Ländern haben kann. Da die JG das Einkommen der JG-TeilnehmerInnen erhöht, ist eine Erhöhung ihrer Konsumausgaben anzunehmen, wodurch letztlich über den fiskalischen Multiplikatoreffekt das Gesamteinkommen und die Gesamtnachfrage steigen. Üblicherweise geht mit höheren Konsum- und Investitionsausgaben einher, dass auch die Anzahl der Importe der aus dem Ausland bezogenen Güter und Dienstleistungen steigt. Wenn die Summe der Importe relativ zu jener der Exporte
IMOMENTUM QUARTERIY "IIIIIIIIIIIIIIIII 235 
steigt, verschiebt das die Handelsbilanz in Richtung Außenhandelsdefizit - je nach Landeskontext kann dies entweder einen verringerten Außenhandelsüberschuss oder ein erhöhtes Außenhandelsdefizit bedeuten. Außenhandelsdefizite können zu einer Abwertung der Währung, also dem äußeren Wert der Währung relativ zu einer ausländischen Währung, führen, da die ausländische Währung - bedingt durch das Faktum, dass die Importe aus dem Ausland die eigenen Exporte übersteigen - stärker nachgefragt wird als die inländische Währung (Wray 2015; Feenstra/Taylor 2014). Eine Abwertung der Währung bedeutet wiederum, dass Importe, also der Kauf von Gütern und Dienstleistungen aus dem Ausland, teurer werden, da nun mehr von der heimischen Währung aufgebracht werden muss, um dem Preis der Importe in ausländischer Währung zu entsprechen. Dieser Preisanstieg wird importierte Inflation genannt (Mitchell et al. 2019).

Hierzu gibt es nun mehrere Punkte, die zu berücksichtigen sind. Zunächst einmal werden Wechselkurse auf dem Finanzmarkt, in dem Fall dem Devisenmarkt, gebildet, die durch Angebot und Nachfrage nach den jeweiligen Währungen zustande kommen. Nun ist es nicht so, dass die Preise, die auf Finanzmärkten zustande kommen, die Verhältnisse der realen Welt widerspiegeln und adäquate Preisinformationen produzieren. Das liegt daran, dass der Großteil der Aktivitäten auf diesen Märkten von Spekulationen und Herdenverhalten getrieben ist (Flassbeck 2018; UNCTAD 2011). Wie Flassbeck (2012) treffend beschreibt, funktioniert der Finanzmarkt gänzlich anders als ein Konsumgütermarkt. Wenn der Preis für Konsumgüter, z. B. für Kartoffeln, steigt, sinkt üblicherweise die Nachfrage. Wenn der Preis für Finanzmarktprodukte, z. B. Aktien oder Währungsderivate, steigt, interpretieren Spekulanten dies als Hinweis, dass es eine Preisrallye geben könnte, die sie natürlich nicht verpassen wollen, sodass die Nachfrage steigt. Der Preismechanismus auf Finanzmärkten funktioniert also fundamental gegensätzlich zu jenem auf Gütermärkten. Es gibt bisher kein ökonomisches Modell, das in der Lage ist, die Veränderungen von Wechselkursen akkurat zu antizipieren (Mitchell et al. 2019). Daher ist letztlich auch der Link von Außenhandelsdefiziten zur Abwertung der Währung nicht verlässlich und bildet keine sichere Grundlage für wirtschaftspolitische Entscheidungen.

Dennoch: Angenommen, es kommt zu dem Effekt, dass die JG-bedingte Einkommenserhöhung zu mehr Konsum und mehr Importen und damit zu einem Außenhandelsdefizit führt, wodurch der Wechselkurs unter Abwertungsdruck gerät und die Möglichkeit von importierter Inflation besteht - wie wäre das $\mathrm{zu}$ beurteilen? Zwar hat eine Abwertung der Währung Verteilungswirkungen im Importland, aber sie gibt keine verlässliche Auskunft über die Veränderung des realen Wohlstandsniveaus. Denn der Wechselkurs determiniert die Kaufkraft einer einzelnen Währungseinheit, aber nicht die Kaufkraft aller Währungseinheiten. Doch genau davon hängt der makroökonomische Effekt für den materiellen Wohlstand eines Landes ab. Für den Gesamtwohlstand ist nicht entscheidend, wie viele Güter und Dienstleistungen mit einem einzelnen Euro (auf jede Währung anwendbar) gekauft werden können, sondern wie viele Güter und Dienstleistungen alle Euros zu kaufen in der Lage sind. Führt also die Vollbeschäftigungspolitik samt Jobgarantie zu einem höheren nominalen Einkommen und dadurch zu mehr Importen, steigt auch der materielle Reichtum des Importlandes, der sich aus der heimischen Produktion zuzüglich aller Importe und abzüglich aller Exporte zusammensetzt. Exporte machen den materiellen Reichtum kleiner, Importe machen den materiellen Reichtum größer. Ein Außenhandelsdefizit, also die Situation, in der die Importe größer als die Exporte sind, vergrößert daher netto den materiellen Wohlstand eines Landes. Umgekehrt reduziert ein Außenhandelsüberschuss den materiellen Wohlstand eines Landes. Eine Währungsabwertung verändert zwar die Kaufkraft einzelner inländischer Akteure, die Gesellschaft insgesamt kann durch die höheren Importe aber materiell bessergestellt sein. Die Verteilungswirkung durch die Veränderung der Kaufkraft im Importland ist durchaus relevant, kann aber ebenfalls und viel effektiver mit der Fiskalpolitik adressiert werden (Wray 2015; Mosler 2012).

Ein übliches Argument für einen Außenhandelsüberschuss ist, dass damit zusätzliche Jobs und Beschäftigung im Inland geschaffen werden. Die MMT argumentiert hingegen: Wenn der originäre Sinn des Wirtschaftens jener ist, die Produkte und Dienstleistungen herzustellen, die wir als Gesellschaft konsumieren möchten, erscheint es erst einmal irrational, Arbeitskraft und Energie in eine Produktion zu stecken, deren Produktionsergebnis letztlich exportiert wird und für die es gegenwärtig keinen Rückfluss an Gütern oder Dienstleistungen gibt - wie es bei einem Exportüberschuss der Fall ist (Hail 2018). Darüber hinaus verliert das Argument, dass Exporte für die Schaffung von Arbeitsplätzen benötigt werden, durch die JG an Relevanz. Die JG schafft stattdessen Jobs, die 
auf die Steigerung des Gemeinwohls ausgerichtet sind (Tcherneva 2020). Hinsichtlich importierter Inflation hilft der Lohnankereffekt der JG dabei, eine womöglich außenwirtschaftlich angefachte Lohn-Preis-Spirale zu dämpfen. Zusätzlich ist der Wert der Währung gekoppelt an die Menge und Qualität der Arbeitskraft, die eine Einheit der Währung kaufen kann, sodass der Wechselkurs letztlich auch widerspiegelt, welche Menge und Qualität an Arbeitskraft eine Einheit der ausländischen Währung nach Umtausch in die inländische Währung erwerben kann, was signifikante, ungerechtfertigte Wechselkursabwertungen $\mathrm{zu}$ vermeiden hilft (Wray 2015). Die Implikationen von importierter Inflation sind für Entwicklungsländer, die häufig zur Deckung ihrer Grundbedürfnisse - in den meisten Fällen Lebensmittel und Energie - auf Importe angewiesen sind, viel bedeutender als für die entwickelten Staaten, da importierte Inflation den Erwerb und letztlich den Zugang zu den lebensnotwendigen Ressourcen erschweren kann (Kaboub 2006). Die Knappheit an Lebensmitteln und Energie ist jedoch ein realwirtschaftliches Problem, das grundlegend auch nur auf dieser Ebene und nicht auf finanzwirtschaftlicher Ebene gelöst werden kann. Die JG ermöglicht, dass alle verfügbaren inländischen Ressourcen - Technologie, Land, Arbeitskraft, Energie - genutzt werden, um bestehende Probleme und Knappheiten auf realwirtschaftlicher Ebene zu lösen. Im Fall von importabhängigen Entwicklungsländern ermöglicht die JG den gezielten Aufbau von alternativen Landwirtschaftsmethoden, z. B. Aquaponik, oder einer Infrastruktur zur Nutzung von erneuerbaren Energien, um die realwirtschaftlichen Problemursachen $\mathrm{zu}$ lösen (Kaboub et al. 2015; Kaboub 2008; Murray/Forstater 2013a). Darüber hinaus ist es ebenso denkbar, die JG auf die Produktion von Gütern für den Export zwecks Kompensation der Handelsbilanzeffekte auszurichten (Kaboub et al 2015). Die bisherige Strategie, also das Nutzen von permanenter Arbeitslosigkeit zum Versuch der Symptommilderung, erscheint in diesem Vergleich dazu aus ökonomischen (und sozialen) Gesichtspunkten unterlegen. Grundsätzlich gilt: Wenn eine Regierung die möglichen Handelsbilanz- und Wechselkurseffekte zu minimieren beabsichtigt, so kann sie jederzeit begleitende wirtschaftspolitische Maßnahmen, wie z. B. Handelspolitik, strategische Importsubstitution bzw. Einfuhrbeschränkungen, Steuerpolitik, Kapitalkontrollen oder Zinspolitik, nutzen (Mitchell et al. 2019). Dies ist eine Frage von politischen Prioritäten.

\section{Zu den sozial-gesellschaftlichen Implikationen \\ einer Jobgarantie}

5.1 Die Kosten von Arbeitslosigkeit

Angesichts der sozialen Krise, die sich beispielsweise in den von hoher Arbeitslosigkeit geplagten südeuropäischen Ländern manifestiert, ist neben den rein ökonomischen Argumenten auch die sozial-gesellschaftliche Komponente einer JG von Relevanz (Eurostat 2020). Schon Martin Luther King Jr. forderte, dass der Staat direkt Beschäftigungsmöglichkeiten kreieren soll und sah die JG als jene Maßnahme, die wie keine andere sozialen und ökonomischen Fortschritt für die systematisch benachteiligte afroamerikanische Gesellschaft brächte. Nicht umsonst war sein bekannter „March on Washington“ offiziell als „March on Washington for Jobs and Freedom" benannt (Forstater 2002).

Mit der JG erlöscht praktisch das Risiko in die Situation unfreiwilliger Arbeitslosigkeit zu geraten, da die JG jeder und jedem, die/der arbeitswillig ist, einen auf das Gemeinwohl ausgerichteten Job $\mathrm{zu}$ sozialverträglichem Lohn inklusive Sozialleistungen anbietet. Dabei gehen die Vorteile der Möglichkeit auf kontinuierliche Beschäftigung weit über die bloße Einkommenssicherheit, die die Deckung der wesentlichen Bedürfnisse sicherstellt, hinaus. Die sozialen Kosten von Arbeitslosigkeit sind größer als die finanziellen (Tcherneva 2017b; Young 2012; Watts/Mitchell 2000; Winkelmann/Winkelmann 1995; McKee-Ryan et al. 2005; Carroll 2007). TeilnehmerInnen des ehemaligen argentinischen JG-Programms („Jefes de Hogar“) stuften, gefragt nach den Gründen für die Zufriedenheit mit dem Programm, „Beschäftigung haben“, „in einem guten Umfeld arbeiten“, „etwas zum Gemeinwohl beisteuern“ sowie „Lernen“ höher als „Einkommen beziehen“ ein (Tcherneva/Wray 2005). Die Option der kontinuierlichen Beschäftigung adressiert die mit unfreiwilliger Arbeitslosigkeit verbundenen sozialgesellschaftlichen Probleme wie Armut, soziale Isolation, Kriminalität, regionale Entwicklungsdisparitäten, Gesundheitsprobleme, Familienprobleme, Schulabbrüche, Verlust von Humankapital sowie allgemeine soziale, politische und wirtschaftliche Instabilität (Mitchell/Muysken 2008; Tcherneva 2020). Darüber hinaus ermöglicht die JG die Realisierung der Vorteile von kontinuierlicher Beschäftigung: Armutsbekämpfung, Stärkung des Gemeinwesens, soziale Vernetzung, Weiterbildung, persönliche Weiterentwicklung sowie den Aufbau von neuen Fertigkeiten. Insbesondere hilft 
die JG denjenigen, die im aktuellen System am meisten benachteiligt sind, z. B. Menschen mit Behinderungen, deren Beschäftigung mit zusätzlichem Aufwand und Kosten einhergeht und die daher nur schwer adäquate Jobs im Privatsektor finden (Wray et al. 2018; Tcherneva 2018). Die JG könnte speziell auf die jeweiligen Bedürfnisse ausgerichtete Jobs schaffen und diesen Bevölkerungsgruppen damit Zugang zu einer würdeund bedeutungsvollen Beschäftigung gewähren - mit all den sozial-psychologischen Vorteilen, wie z. B. sozialer Integration (Modini et al. 2016). Letztlich ist die JG die Umsetzung des bereits in der Allgemeinen Erklärung der Menschenrechte verankerten Rechts, welches im neoliberalen Paradigma durch die permanente Existenz von unfreiwilliger Arbeitslosigkeit vernachlässigt wurde:

„1. Jeder Mensch hat das Recht auf Arbeit, auf freie Berufswahl, auf gerechte und befriedigende Arbeitsbedingungen sowie auf Schutz vor Arbeitslosigkeit. 2. Jeder Mensch, ohne Unterschied, hat das Recht auf gleichen Lohn für gleiche Arbeit." (Artikel 23, Allgemeine Erklärung der Menschenrechte)

\subsection{Die Arbeitsbedingungen der Jobgarantie als effektive Untergrenze}

Der in der JG bezahlte Lohn wird gleichzeitig zum effektiven nationalen Mindestlohn. Während aktuelle Mindestlöhne nur für diejenigen, die im Privatsektor bzw. im regulären öffentlichen Sektor eine Beschäftigung finden, gelten, gilt der Mindestlohn nicht für diejenigen, die aktuell von unfreiwilliger Arbeitslosigkeit betroffen sind, aber bereit wären, zum Mindestlohn zu arbeiten - diese erhalten lediglich das Arbeitslosengeld, welches in vielen Fällen ein Monatseinkommen unterhalb des Mindestlohnes bedeutet. Im Grunde macht die JG das Einführen sowie das Administrieren von Mindestlohngesetzen überflüssig, wenn abhängig Beschäftigte eine besser bezahlte und örtlich verfügbare Jobalternative haben (Tcherneva 2012). Der Privatsektor wird also zur Rekrutierung von Arbeitskräften ein besseres Angebot als jenes in der JG machen müssen. Das bedeutet, dass die JG eine effektive Untergrenze an akzeptablen Arbeitsbedingungen in den Arbeitsmarkt einzieht und somit auch ein Mittel ist, um eine Verbesserung der Arbeitsbedingungen - von Lohn über nichtgeldliche Leistungen bis hin zur Arbeitsatmosphäre - im Privatsektor $\mathrm{zu}$ erwirken. Gleichermaßen gilt dies auch für den informellen Sektor, der z. B. in Entwicklungsländern tendenziell größer ausgeprägt ist (Murray/Forstater 2013a; Günther/Launov 2012).

In der aktuellen Situation, in der unfreiwillige Arbeitslosigkeit permanent existiert, sind die Kräfteverteilungen und Verhandlungspositionen auf dem Arbeitsmarkt asymmetrisch zugunsten der Arbeitgeberseite verteilt. Nicht zuletzt geht das Zeitalter des Neoliberalismus auch mit einer Schwächung der Gewerkschaftszugehörigkeit einher und hat so den Organisationsgrad der ArbeitnehmerInnen verringert, was deren kollektive Verhandlungsmacht einschränkt (Mitchell/Muysken 2008). Diejenigen mit einer vergleichsweisen geringen Arbeitsqualifikation sind jene ArbeitnehmerInnen, die im Boom als Letzte Anstellung finden und im Abschwung als Erstes entlassen werden - also diejenigen mit der geringsten Verhandlungsmacht. Folglich ist es gerade diese Personengruppe, die aufgrund der asymmetrischen Verhandlungsmacht in Situationen gerät, in denen sie eigentlich nicht zu akzeptierende Arbeitsbedingungen akzeptiert bzw. akzeptieren „muss“ (Tcherneva 2013). Für diejenigen, die lediglich im informellen Sektor Beschäftigungsmöglichkeiten finden, ist die Situation gar noch schlechter, da sie dort gänzlich ungeschützt der Machtasymmetrie ausgesetzt sind. Das ist besonders relevant für Entwicklungsländer, in denen unter Umständen auch das Justizsystem noch nicht ausreichend entwickelt ist (Günther/Launov 2012; Kaboub 2006). Die JG gibt diesen Personengruppen die Chance, inakzeptablen Arbeitsbedingungen $\mathrm{zu}$ entsagen und adressiert damit die asymmetrischen Machtverhältnisse auf dem Arbeitsmarkt.

\subsection{Die Demokratierelevanz der Jobgarantie}

Darüber hinaus hat die JG demokratierelevante Implikationen. Das zentrale Ideal der Demokratie kann vereinfacht so verstanden werden, dass den BürgerInnen eine ausgewogene Teilhabe in den wesentlichen Entscheidungen, die Einfluss auf das Leben bzw. das Lebensumfeld nehmen, ermöglicht wird. In Anbetracht dessen, wie viel Zeit Menschen in ihrem Arbeitsumfeld verbringen, ist die Abwesenheit von demokratischen Strukturen am Arbeitsplatz als demokratisches Versäumnis zu interpretieren. Der Bottom-up-Ansatz der JG und das Faktum, dass die Jobs auf die Verbesserung des örtlichen Gemeinwohls ausgerichtet sind, indem sie etwa über Gemeindeumfragen identifizierte Bedarfe adressieren, kann das Teilhabeempfinden der BürgerInnen stärken (Tcherneva 2020; Tcherneva 2013; 
Ehnts/Höfgen 2019a). Dazu kann die Implementierung von demokratischen Strukturen innerhalb der JG ein Hebel sein, um diese auch im Privatsektor zu implementieren (Tcherneva 2012). Darüber hinaus ist das bestehende Level an Einkommens- und Vermögensungleichheit eine Bürde für die Demokratie, da es bestimmten Personengruppen ermöglicht, überverhältnismäßig großen Einfluss zu nehmen. Da die JG den am meisten Benachteiligten am unteren Ende der Einkommensverteilung einen Job sowie eine Einkommenssteigerung verschafft, beeinflusst die JG die Einkommensungleichheit positiv und ist damit eines der vielen Puzzlestücke, die zu einer Korrektur der Ungleichheit beitragen können (Ehnts/Höfgen 2019a).

\section{Fazit}

Die Denkschule der MMT ermöglicht ein grundlegend anderes Verständnis des Geldsystems und der monetären Zusammenhänge der Wirtschaft. Ein Verständnis, das auf den institutionellen Gegebenheiten und auf der Logik der doppelten Buchführung, eine in der MMT häufig genutzten Methode zur Darstellung der monetären Zusammenhänge, fußt. Kerninhalt der MMT ist zum einen die Auffassung, dass ein wichtiger Zweck des Geldsystems die Ermöglichung der optimalen Bewirtschaftung der verfügbaren realen Ressourcen ist, und zum anderen die Einsicht, dass die staatliche Währung im Zentrum des Geldsystems steht. Daraus ergibt sich, dass ein Staat mit einem hohen Grad an monetärer Souveränität unter gänzlich anderen ökonomischen Voraussetzungen agiert als ein Staat, dem lediglich ein geringer Grad an monetärer Souveränität zugeschrieben werden kann. Gleiches trifft auf währungsnutzende Wirtschafssubjekte zu, die innerhalb anderer ökonomischer Restriktionen agieren als währungsherausgebende Institutionen. Die Analogie zwischen Staat und Privathaushalt ist demnach irreführend. Aus Sicht der MMT sollte die Wirtschaftspolitik des Staates daher anhand anderer Parameter bewertet werden. Das bedeutet konkret, dass bloße Finanzgrößen, wie das staatliche Defizit oder auch der Stand der Staatsschulden, durch funktionelle Parameter, wie etwa die Höhe der unfreiwilligen Arbeitslosigkeit, als Bewertungskriterien für die Wirtschaftspolitik ersetzt werden sollten. Ferner ist daraus abzuleiten, dass die Höhe der unfreiwilligen Arbeitslosigkeit Ausdruck für zu geringe Nettoausgaben des Staates und damit letztlich eine wirtschaftspolitische Entscheidung des Staates ist. Durch eine JG ist der Staat in der Lage, die fiskalischen
Ausgaben in der Höhe automatisch so anzupassen, dass dauerhafte Vollbeschäftigung erreicht wird.

Die JG als wirtschaftspolitische Reform hat das Potenzial, auf vielfältige Weise zur makroökonomischen Stabilisierung und zu sozial-gesellschaftlichem Fortschritt beizutragen. Während der Werkzeugkasten des ökonomischen Mainstreams nicht ohne die permanente Existenz unfreiwilliger Arbeitslosigkeit auskommt - und diese daher schlicht als natürliche Arbeitslosenquote oder individuelle Präferenzentscheidungen deklariert -, bietet der Werkzeugkasten der MMT eine Alternative zur Erreichung von Vollbeschäftigung und Preisstabilität an, die zudem noch die gesellschaftlichen Kosten von Arbeitslosigkeit adressiert. Der Artikel hat gezeigt, dass die JG aus rein makroökonomischer, aber auch aus verschiedenen politischen Perspektiven vertreten werden kann. Angesichts der großen gesellschaftlichen Herausforderungen bietet sich die JG in ihrer Umfänglichkeit als integraler Bestandteil eines notwendigen Strukturwandels im Sinne des ökologischen Umbaus der Wirtschaft an. Dementsprechend wird die JG etwa als Teil eines Green New Deal diskutiert.

Wie bei jeder wirtschaftspolitischen Reform hängt die Realisierung der potenziellen Vorteile von der Qualität der Implementierung und den institutionellen Rahmenbedingungen ab. Das betrifft ganz konkret die Frage, wie das Design der JG in der Praxis ausgestaltet wird. Darüber hinaus sollte die JG auch nicht als Allheilmittel für makroökonomische Stabilisierungspolitik und arbeitsmarktpolitische Regulierung verstanden werden. Sie ist eines von vielen geeigneten Instrumenten, um die wirtschaftspolitischen Ziele zu erreichen. Aber sie ist explizit kein Ersatz für diskretionäre Fiskalpolitik, für ein gemeinwohlorientiertes Steuersystem, für eine angemessene Lohnpolitik oder für eine innovationsfördernde Industriepolitik.

Die Autoren identifizieren über die in diesem Beitrag aufgeführten Gesichtspunkte hinaus weiteren Forschungsbedarf. Bei Anwendung der Analyse auf einen institutionellen Kontext, etwa bei einer etwaigen Einführung in der Eurozone, bedarf es konkreter Untersuchungen mit Blick auf die konjunkturpolitischen und arbeitsmarktpolitischen Auswirkungen. Ebenso ist die institutionelle Ausgestaltung des Geldsystems, wie erwähnt, ein wichtiger Faktor. Ein Faktor, der maßgeblich von politischen Erwägungen abhängt, die daher auch im jeweiligen Kontext berücksichtigt werden müssen. Gäbe es beispielsweise unter den Regierungen der Euroländer einen politischen Konsens für QUARTERLY IIIIIIIIIIIIIIIII 239 
eine tiefergehende Integration, wäre die Ausgestaltung dieser Integration, z. B. in Form eines „Euro-Treasury“, sowie deren Konsequenzen für das Design einer JG zu reflektieren. Gleiches gilt für den Fall, wenn die JG in einem Land der Eurozone unter den derzeit gegebenen Rahmenbedingungen eingeführt werden sollte und damit gegebenenfalls Abwägungen und Kompromisse mit anderen politischen Maßnahmen erfordern würde. Angesichts der interdisziplinären Facetten würde die JG ferner durch weitere Forschung aus den Bereichen der Politökonomie, der Soziologie und der Psychologie profitieren.

\section{Literatur}

AMS Arbeitsmarktservice (2020): Arbeitsmarktdaten im Juni 2020. Wien.

Bell, S. (2000): Do taxes and bonds finance government spending? Journal of Economic Issues, 34 (3), 603-620.

Bell, S. (2001): The role of the state and the hierarchy of money. Cambridge Journal of Economics, 25 (2), 149-163.

Bell, S./Wray, L. R. (2002): Fiscal Effects on Reserves and the Independence of the Fed. Journal of Post Keynesian Economics, 25 (2), 263-271.

Bundesagentur für Arbeit (2020): Der Arbeitsmarkt im Mai 2020. https://www.arbeitsagentur.de/presse/202032-der-arbeitsmarkt-im-mai-2020

Carroll, N. (2007): Unemployment and psychological wellbeing. Economic Record, 83 (262), 287-302.

Cruz-Hidalgo, E./Ehnts, D. H./Tcherneva, P. R. (2019): Completing the Euro: The Euro Treasury and the Job Guarantee. Revista de Economia Critica, 27(1), 100-111.

Ehnts, D. H. (2020a): The fiscal-monetary nexus in Germany. Working Paper.

Ehnts, D. H. (2020b): Geld und Kredit: eine €-päische Perspektive. Marburg: Metropolis Verlag.

Ehnts, D. H./Höfgen, M. (2019a): The Job Guarantee: Full Employment, Price Stability and Social Progress. Society Register, 3 (2), 49-65.

Ehnts, D. H./Höfgen, M. (2019b): Modern monetary theory: a European perspective. real-world economics review, 89, 75-84.

Ehnts, D. H./Höfgen, M. (2020): Modern Monetary Theory and the Public Purpose. American Review of Political Economy, 15(1), 1-12

Ehnts, D. H./Paetz, M. (2019): Die Modern Monetary Theory: Staatsschulden als Steuergutschriften. Vierteljahrshefte zur Wirtschaftsforschung/Quarterly Journal of Economic Research, 88 (4), 77-89.

EUROSTAT (2020): Unemployment Statistics June 2020. Online: https://ec.europa.eu/eurostat/statistics-explained/
index.php/Unemployment_statistics\#Youth_unemployment [20.10.2020]

Feenstra, R. C./Taylor, A. M. (2014): International Macroeconomics. New York: Worth Publishers.

Flassbeck, H. (2012): Die Marktwirtschaft des 21. Jahrhunderts. Frankfurt am Main: Westend Verlag.

Flassbeck, H. (2018): Exchange rate determination and the flaws of mainstream monetary theory. Brazilian Journal of Political Economy, 38 (1), 99-114.

Forstater, M. (2011): „Jobs for all“: Another dream of the rev. Dr. Martin Luther King, Jr. Forum for Social Economics, $31(2), 45-53$.

Forstater, M. (2005): Taxation and primitive accumulation: the case of colonial Africa. Research in Political Econo$m y, 22$ (1), 51-64.

Forstater, M./Mosler, W. (2005): The natural rate of interest is zero. Journal of economic issues, 39 (2), 535-542.

Fullbrook, E./Morgan, J. (2020): Modern monetary theory and its critics. Bristol: World Economics Association Books.

Fullwiler, S./Grey, R./Tankus, N. (2019): An MMT response on what causes inflation. Financial Times. Online: https://www.ft.com/content/539618f8-b88c-3125-8o31-cf46ca197c64 [20.10.2020].

Fullwiler, S. T. (2006): Setting interest rates in the modern money era. Journal of Post Keynesian Economics, 28 (3), 496-525.

Fullwiler, S. T. (2008): Modern Central Bank operations: the general principles. In: Rochon, L.-Ph./Rossi, S. (Hg.): Advances in Endogenous Money Analysis. Cheltenham: Edward Elgar Publishing, 50-87.

Fullwiler, S. T. (2010): Modern monetary theory-a primer on the operational realities of the monetary system. Available at SSRN 1723198.

Fullwiler, S. T. (2011): Treasury Debt Operations: An analysis integrating social fabric matrix and social accounting matrix methodologies. Available at SSRN 1825303.

Fullwiler, S. T./Bell, S./Wray, L. R. (2012): Modern Money Theory: a response to critics. Available at SSRN 2008542.

Galbraith, J. K. (1997): Time to Ditch the NAIRU. Journal of Economic Perspectives, 11 (1), 93-108.

Günther, I./Launov, A. (2012): Informal employment in developing countries: Opportunity or last resort? Journal of development economics, 97 (1), 88-98.

Hail, S. (2018): Economics for Sustainable Prosperity. Berlin/ Heidelberg: Springer.

Höfgen, M. (2020): Mythos Geldknappheit: Modern Monetary Theory oder warum es am Geld nicht scheitern muss. Stuttgart: Schaeffer Poeschel Verlag.

Innes, M. (1914): The credit theory of money. Banking Law Journal, 31, 151-168.

Institut Arbeit und Qualifikation der Universität DuisburgEssen (2019): Arbeitslose und gemeldete offene Arbeitsstellen, 2000-2019. 
Kaboub, F. (2006): A roadmap to full employment and price stability in developing countries: The case of Tunisia, Ann Arbor, Mich.: UMI

Kaboub, F. (2008): Elements of a radical counter-movement to neoliberalism: Employment-led development. Review of Radical Political Economics, 40 (3), 220-227.

Kaboub, F./Forstater, M./Kelsay, M. (2015): The cost of unemployment and the job guarantee alternative in Saudi Arabia. Policy report, 101.

Kelton, S. (2011): Limitations of the government budget constraint: Users vs. issuers of the currency. Panoeconomicus, $58(1), 57-66$.

Kelton, S. (2020): The deficit myth: modern monetary theory and the birth of the people's economy. New York: Public Affairs.

Knapp, G. F. (1905): Staatliche Theorie des Geldes. München: Duncker \& Humblot.

Mckee-Ryan, F./Song, Z./Wanberg, C. R./Kinicki, A. J. (2005): Psychological and physical well-being during unemployment: a meta-analytic study. Journal of applied psychology, $90(1), 53-76$.

Mitchell, W./Fazi, T. (2017): Reclaiming the state. A Progressive Vision of Sovereignty for a Post-Neoliberal World. University of Chicago Press Economics Books.

Mitchell, W./Muysken, J. (2008): Full employment abandoned: shifting sands and policy failures. Cheltenham: Edward Elgar Publishing.

Mitchell, W./Wray, L. R./Watts, M. (2019): Macroeconomics. London: Red Globe Press.

Modini, M./Joyce, S./Mykletun, A./Christensen, H./Bryant, R. A./Mitchell, P. B./Harvey, S. B. (2016): The mental health benefits of employment: Results of a systematic meta-review. Australasian Psychiatry, 24 (4), 331-336.

Mosler, W. (1997): Full employment and price stability. Journal of Post Keynesian Economics, 20 (2), 167-182.

Mosler, W. (1998): Exchange rate policy and full employment. The University of Newcastle, Australia.

Mosler, W. (2010): Seven deadly innocent frauds of economic policy. Valance Co., Inc.

Mosler, W. (2012): Soft Currency Economics II: The Origin of Modern Monetary Theory. CreateSpace Independent Publishing Platform.

Mosler, W./Forstater, M. (1999): A general framework for the analysis of currencies and commodities. In: Davidson, P./Kregel, J. (Hg.): Full Employment and Price Stability in a Global Economy. Cheltenham: Edward Elgar Publishing, 166-177.

Mosler, W./Silipo, D. B. (2017): Maximizing price stability in a monetary economy. Journal of Policy Modeling, 39 (2), 272-289.

Murray, M./Forstater, M. (2013a): Employment guarantee schemes: job creation and policy in developing countries and emerging markets. Berlin/Heidelberg: Springer.

Murray, M./Forstater, M. (2013b): The job guarantee: Toward true full employment. Berlin/Heidelberg: Springer.
Nersisyan, Y./Wray, L. R. (2016): Modern Money Theory and the facts of experience. Cambridge Journal of Economics, 40 (5), 1297-1316.

Palley, T. I. (2005): From Keynesianism to neoliberalism: Shifting paradigms in economics. In: Saad-Filho, A./ Johnston, D. (Hg.): Neoliberalism: A critical reader. London, Pluto Press, 20-29.

Ruml, B. (1946): Taxes for revenue are obsolete. American Affairs, 8 (1), 35-39.

Springer Gabler. (2018). Arbeitslosigkeit. https://wirtschaftslexikon.gabler.de/definition/arbeitslosigkeit-27801/version-327668 [20.10.2020].

Tamesberger, D./Theurl, S. (2019): Vorschlag für eine Jobgarantie für Langzeitarbeitslose in Österreich. ICAE Working Paper Series.

Tcherneva, P. R. (2002): Monopoly money: The state as a price setter. Oeconomicus, 5 (Winter), 30-52.

Tcherneva, P. R. (2006): Chartalism and the tax-driven approach to money. In: Arestis, Ph./Sawyer, M. (Hg): A handbook of alternative monetary economics. Cheltenham: Edward Elgar Publishing, 69-87.

Tcherneva, P. R. (2012): Beyond full employment: The employer of last resort as an institution for change. The Levy Economics Institute's Working Paper Series, Working Paper Nr. 732

Tcherneva, P. R. (2013): The job guarantee: delivering the benefits that basic income only promises-a response to guy standing. Basic Income Studies, 7 (2), 66-87.

Tcherneva, P. R. (2017a): Money, power and distribution: implications for different monetary regimes. Journal of Self-Governance and Management Economics, 5 (3), 7-27.

Tcherneva, P. R. (2017b): Unemployment: the silent epidemic. The Levy Economics Institute's Working Paper Series, Working Paper Nr. 895.

Tcherneva, P. R. (2018): The Job Guarantee: Design, Jobs, and Implementation. The Levy Economics Institute's Working Paper Series, Working Paper Nr. 902.

Tcherneva, P. R. (2020): The Case for a Job Guarantee. Hoboken: John Wiley \& Sons.

Tcherneva, P. R./Wray, L. R. (2005): Gender and the Job Guarantee: The Impact of Argentina's Jefes Program on Female Heads of Poor Households. Available at SSRN 1009594.

Tymoigne, E. (2014): Modern Money Theory, and Interrelations Between the Treasury and Central Bank: The Case of the United States. Journal of Economic Issues, 48 (3), 641-662.

Tymoigne, E. (2019): Monetary Sovereignty: Nature, Implementation, and Implications. Public Budgeting \& Finance, 40 (3), 49-71.

Tymoigne, E./Wray, L. R. (2015): Modern money theory: a reply to Palley. Review of political economy, 27 (1), 24-44.

UNCTAD (2011): Trade and Development Report. United Nations Conference on Trade and Development. Online: https://unctad.org/en/Docs/tdr2011_en.pdf [20.10.2020]. 
Watts, M. J./Mitchell, W. F. (2000): The costs of unemployment in Australia. London: Sage Publications Sage UK.

Winkelmann, L./Winkelmann, R. (1995): Unemployment: where does it hurt?. CEPR Discussion Papers No 1093

Wray, L. R. (1997): Government as employer of last resort: full employment without inflation. The Levy Economics Institute's Working Paper Series, Working Paper Nr. 213.

Wray, L. R. (2014): From the state theory of money to modern money theory: An alternative to economic orthodoxy. The Levy Economics Institute's Working Paper Series, Working Paper Nr. 792.

Wray, L. R. (2015): Modern money theory: A primer on macroeconomics for sovereign monetary systems. Berlin/Heidelberg: Springer.

Wray, L. R./Dantas, F./Fullwiler, S./Tcherneva, P. R./Kelton, S. A. (2018): Public service employment: A path to full employment. Research Project Report. Annandale-onHudson, NY: Levy Economics Institute of Bard College, April.

Wray, L. R./Sardoni, C. (2007): Fixed and Flexible Exchange Rates and Currency Sovereignty. The Levy Economics Institute's Working Paper Series, Working Paper Nr. 489.

WSJ Editorial Board (2020): A Trillion Here, a Trillion There. Wall Street Journal, 8.5.2020.

Young, C. (2012): Losing a job: The nonpecuniary cost of unemployment in the United States. Social Forces, 91 (2), 609-634. 